\title{
Cronología de la regeneración del bosque tropical seco en Santa Rosa, Guanacaste, Costa Rica. I. Características edáficas
}

\author{
Jorge A. Leiva ${ }^{1}$, Rafael Mata ${ }^{1}$, Oscar J. Rocha ${ }^{2} \&$ Marco V. Gutiérrez Soto ${ }^{3 *}$ \\ 1. Universidad de Costa Rica, Centro de Investigaciones Agronómicas, 11503-2060, San José, Costa Rica; \\ jorge.leivasanabria@ucr.ac.cr \\ 2. Department of Biological Sciences, Kent State University, Ohio, USA; orocha@kent.edu \\ 3. Universidad de Costa Rica, Estación Experimental Fabio Baudrit, Apdo. 183-4050 Alajuela, Costa Rica. Tel: (506) \\ 2433-9111. Fax: (506) 2433-9086; surdo26@racsa.co.cr \\ * Correspondencia
}

Recibido 15-V-2008. Corregido 20-II-2009. Aceptado 25-III-2009.

\begin{abstract}
Chronology of tropical dry forest regeneration in Santa Rosa, Guanacaste, Costa Rica. I. Edaphic characteristics. We characterized soil chemical and physical properties in eight tropical dry forest (TDF) successional sites along a time sequence (10, 15, 20, 40, 60 and >100 years) in Santa Rosa, Costa Rica. Seventeen soils were identified, described, and classified in six orders. Most soils were classified as Entisols and Vertisols, but Mollisols, Alfisols, Inceptisols and Ultisols were also present. All soils showed good fertility that did not constrain species richness. High edaphic variation seemed the result of complex interactions among the pyroclastic origin of Santa Rosa ignimbritic plateau (SRIP), the lithological composition and acidity of ignimbritic rocks, the strong seasonality in rainfall, intense hydric and aeolic erosion, topographic variations within the SRIP, and past human intervention. Correlations performed on soils classified as Entisols $(<60 \mathrm{~cm}$ deep) showed a depletion of soil cations (Ca, Mg, K, Na and CEC) during the first 20 years of forest regeneration, that later recovered in mature TDF sites. Organic matter content did not change significantly along the TDF chrono-sequence. Substantial increments in macro-pores and soil hydraulic conductivity were observed, probably resulting from higher root biomass and turnover in older successional sites. Soil available water and meso-pore abundance were negatively correlated with TDF successional age. Our results indicate that edaphic chan ges observed along TDF regeneration might have been due to annual fires in pastures and young TDF sites, addition of decaying litter and fine roots as regeneration progressed, milder microclimate conditions during late regeneration in mature TDF sites, increased nutrient cycling, and the predominance of sandy loam textures among the soils examined. These changes in the soil environment with succession may have physiological and phenological consequences on the species appearing at different stages of TDF regeneration. Rev. Biol. Trop. 57 (3): 801-815. Epub 2009 September, 30.
\end{abstract}

Keywords: Santa Rosa National Park, soils, chemical properties, physical properties, seasonally dry tropical forests, pastures, forest succession.

Los bosques tropicales estacionalmente secos (BTS) de Meso América contienen una alta riqueza estructural y funcional (Murphy \& Lugo 1986, Medina 1995), proveen numerosos servicios ambientales (Maas et al. 2005), y constituyen un modelo para la restauración ecológica y el estudio de la regeneración de los ecosistemas tropicales (Janzen 2000). Estas zonas de vida han sido las predilectas para el desarrollo de importantes asentamientos humanos a través del tiempo, lo que ha causado enormes perturbaciones ecológicas; los BTS son actualmente un mosaico de fragmentos de bosques sucesionales secundarios (Kramer 1997). En la actualidad, los BTS continúan sometidos a presiones superiores a las enfrentadas por sus contrapartes, los bosques tropicales lluviosos (Janzen 1986), y el éxito de los esfuerzos para 
restaurarlos dependerá en gran medida del conocimiento de los procesos sucesionales, el cual es considerablemente menor que el existente sobre los bosques templados (Bazzas 1979) y los ecosistemas tropicales lluviosos (Bazzaz \& Pickett 1980, Mulkey et al. 1996).

En contraste con los ecosistemas húmedos poco estacionales, donde la sucesión ecológica es controlada en gran medida por la dinámica de los claros y el banco de semillas (Guariguata \& Ostertag 2001), los BTS son afectados por perturbaciones ambientales severas, como la incidencia estacional del fuego y la sequía (Ewel 1980). La combinación de factores edáficos, la historia previa del uso de la tierra, la influencia de la vegetación remanente, y la impredecibilidad de la sequía y el fuego, se combinan para producir múltiples rutas sucesionales (Ewel 1980, Mesquita et al. 2001, Chazdon 2003). Entre estos factores, las variaciones edáficas locales y sus efectos sobre la trayectoria sucesional han recibido la menor atención.

La regeneración de los BTS en esta parte de Costa Rica ocurre principalmente sobre la Meseta Ignimbrítica de Santa Rosa (MISR), una región de subducción de placas con constante actividad volcánica, con relieve montañoso y suelos meteorizados, de perfiles poco profundos pero con horizontes conspicuos. El paisaje actual de la región está dominado por potreros ganaderos quemados anualmente, predios abandonados, fragmentos de bosques secundarios y bosques maduros. La MISR tiene una extensión de 1 500-1 $600 \mathrm{~km}^{2}$, un volumen aproximado de roca densa de $100-140 \mathrm{~km}^{3}$ y está compuesta por ignimbritas (tobas), depósitos sedimentarios y coladas de lava (Gillot et al. 1994, Kussmaul 2000). La génesis de los suelos de Santa Rosa ha ocurrido sobre un material parental cuya deposición y formación se inició hace aproximadamente ocho millones de años, como resultado de flujos piroclásticos provenientes de la Cordillera Volcánica de Guanacaste (Kussmaul 2000). La pedogénesis heterogénea en Santa Rosa y sitios aledaños ha sido reconocida en estudios anteriores (Mata 1982), y relacionada con la irregularidad en la consolidación de estos flujos piroclásticos y de la composición química de las rocas. La heterogeneidad en la temperatura de consolidación de los flujos piroclásticos generó una gran variación en la composición de las rocas ignimbríticas y su susceptibilidad a la meteorización (Chiesa 1991, Winters 1997).

La predominancia de perfiles de poca profundidad es resultado del carácter ácido de las rocas ignimbríticas. Los altos contenidos de feldespatos de potasio y tectosilicatos (cuarzo, albita, anortita, ortoclasa) hacen que la meteorización geoquímica sea más difícil, en comparación con rocas máficas o de mayor contenido de cationes, como los basaltos y las dioritas (Buol et al. 1989). Además, la pronunciada estacionalidad climática (5-6 meses secos) reduce la reactividad geoquímica y la meteorización de los minerales primarios de las rocas (Buol et al. 1989). Otros agentes importantes en la formación de los suelos de la MISR han sido el impacto de la erosión eólica e hídrica durante las estaciones seca y lluviosa, respectivamente, las diferencias en el drenaje, y la consecuente disminución o acumulación de nutrientes (Mata 1982). Además, la sustitución del BTS por repastos ganaderos con especies exóticas ha generado prescripción anual de fuego, compactación, y la significativa pedoturbación o mezcla del suelo.

Estudios recientes de la regeneración de los BTS indican que la recuperación de la estructura y las funciones del bosque se alcanza en plazos relativamente cortos (Guariguata \& Ostertag 2001, Chazdon 2003, Ruiz et al. 2005). Los estudios de las relaciones sueloplanta durante la regeneración boscosa tropical son muy escasos (Lathwell \& Grove 1986, Clark 2002). Las variaciones edáficas locales y sus efectos sobre la ruta sucesional han recibido poca atención, aunque varios estudios indican que el suelo influencia la estructura, la diversidad y la abundancia de muchas especies de árboles tropicales (Huston 1980, Clark et al. 1999). Asimismo, los efectos de la vegetación sobre el suelo son variados e incluyen cambios en $\mathrm{pH}$, capacidad de intercambio catiónico y bases intercambiables (Zinke 1962), cantidad 
y calidad de la materia orgánica, y contenido de nitrógeno (Russell et al. 2007). Estudios realizados en otros BTS cercanos a Santa Rosa indican que conforme aumenta la edad de abandono del sitio, las especies arbóreas pioneras sustituyen el componente herbáceo, aumenta la adición anual de hojarasca y raíces finas, se conserva mejor la humedad y se enriquece la superficie del suelo con cationes y materia orgánica (Alfaro et al. 2001).

Este trabajo documenta las influencias edáficas sobre la regeneración boscosa y la vegetación durante la sucesión del BTS. En el primer artículo de una serie de dos, describimos las propiedades de los suelos a lo largo de una cronosecuencia sucesional del BTS de Santa Rosa, Costa Rica $(10,15,20,40,60$ y $>100$ años) y examinamos las interacciones entre las propiedades edáficas y la regeneración del BTS. Los objetivos de este estudio fueron caracterizar la variación edáfica local mediante la medición de las propiedades químicas, físicas y orgánicas de los suelos asociados a la sucesión del BTS, y correlacionar esta variación con la regeneración boscosa.

\section{MATERIALES Y MÉTODOS}

Localización y cronosecuencia de la regeneración boscosa: Este estudio se realizó en el Parque Nacional Santa Rosa (PNSR, $10^{\circ} 50^{\prime} \mathrm{N}, 85^{\circ} \mathrm{W}$ ) y en la Hacienda Pelón de la Altura (HPA, $10^{\circ} 51^{\prime} \mathrm{N}, 85^{\circ} 33^{\prime} \mathrm{W}$ ), en Liberia, Guanacaste, entre agosto del 2002 y julio del 2003. Las zonas de vida dominantes en los sitios de estudio son el bosque seco tropical y el bosque húmedo tropical transición a basal, con un clima estacional, una estación seca de 3.5-6.5 meses, usualmente entre noviembre y abril. La precipitación anual varía entre 1 500 y $1950 \mathrm{~mm}$, la temperatura media anual oscila entre 24.0 y $27.8{ }^{\circ} \mathrm{C}$, y la relación entre evapotranspiración y precipitación es cercana a 1 (Holdridge 1978). Se estableció una cronosecuencia de ocho sitios boscosos de seis edades aproximadas de sucesión $(10,15,20,40,60$ y $>100$ años) desde el abandono del sitio o última quema de pasturas ganaderas. Las edades de los sitios fueron obtenidas de los registros del PNSR y la HPA.

Establecimiento de las parcelas: Se establecieron 130 parcelas de muestreo de $10 \times 50$ $\mathrm{m}\left(500 \mathrm{~m}^{2}\right.$ o $\left.0.05 \mathrm{ha}\right)$ en un área total de 6.5 ha, con orientación norte. Se establecieron 100 parcelas en cinco bosques secundarios $(10,15$, 20,40 y 60 años) (20 por sitio) y 30 parcelas en tres bosques antiguos ( $>100$ años) (10 por sitio). En los sitios de relieve plano (pendientes $<5 \%$; bosques de 10, 15 y 20 años), las parcelas se establecieron en filas y separadas por $20 \mathrm{~m}$. En los sitios ondulados (5-30\% de pendiente), las parcelas representaron el relieve: cima, pendiente y piedemonte. Se consideró como bosque secundario la vegetación establecida sobre sitios abandonados, anteriormente repastos ganaderos dominados por pastos exóticos que propician el fuego (Daubenmire 1972). Los bosques maduros correspondieron a fragmentos con una edad $>100$ años.

Descripción morfológica de los suelos: Se describió el perfil del suelo según el protocolo del Departamento de Agricultura de Estados Unidos (Soil Survey Staff 2007). Se describió la localización, fisiografía, elevación, y la pendiente del paisaje circundante. El perfil del suelo fue muestreado con un barreno holandés, en un sitio elegido al azar en dos coordenadas asociadas a las dimensiones de la parcela. Se midió la fertilidad aparente, el drenaje, la pedregosidad, la erosión y la localización del manto freático. Se identificaron los horizontes del perfil según su profundidad, color y textura. Este protocolo fue completado mediante extracciones de 20 muestras de suelo/ha, que permitió reconocer los suelos dominantes o clases modales dentro de cada bosque. Entre agosto 2003 y marzo 2004 se describieron las clases modales en sitios representativos, tomando muestras de cada horizonte hasta la mayor profundidad posible. Las clases de suelos fueron clasificados a nivel de subgrupo según el sistema del Soil Survey Staff (2007).

Características químicas: Los análisis químicos y físicos de los suelos se realizaron 
en el Centro de Investigaciones Agronómicas de la Universidad de Costa Rica. Las muestras de suelo se secaron en un horno durante 24 horas a $105^{\circ} \mathrm{C}$, se molieron y pasaron por un tamiz de $2 \mathrm{~mm}$. En muestras tomadas de cada horizonte y mantenidas en bolsas plásticas, se midió el $\mathrm{pH}$ con un potenciómetro en una relación suelo y agua de 1:2.5. Se midió el contenido de bases extraíbles $(\mathrm{Ca}, \mathrm{Mg}, \mathrm{K}$ y Na$)$ y la capacidad de intercambio catiónico (CIC) extraídos con acetato de amonio $1 \mathrm{M}(\mathrm{pH} 7)$ y determinados por absorción atómica, todos expresados en cmol $(+) \mathrm{kg} / \mathrm{suelo}$. En suelos con cristales amorfos detectados en el campo mediante la prueba de NaF (Fields y Perrot 1966), se tomaron muestras de cada horizonte hasta $60 \mathrm{~cm}$ de profundidad. En ellas se midió el contenido de $\mathrm{Fe}$ y $\mathrm{Al}$ amorfos extraíbles en oxalato de amonio $0.2 \mathrm{M}(\mathrm{pH} 3.5)$ y determinados mediante absorción atómica. El contenido de materia orgánica se determinó mediante la técnica Walkley-Black, con digestión húmeda en ácido sulfúrico y $\mathrm{K}_{2} \mathrm{Cr}_{2} \mathrm{O}_{7}$, determinando el dicromato de potasio no oxidado, por titulación con sulfato amónico ferroso (Nelson \& Sommers 1982).

Características físicas: Se tomaron muestras de todos los horizontes en bolsas plásticas y en cilindros de acero inoxidable de $4.78 \mathrm{~cm}$ de diámetro. En los cilindros $\left(134.6 \mathrm{~cm}^{3}\right)$ se midió la conductividad hidráulica $\left(k_{h}, \mathrm{cmh}^{-1}\right)$ y la densidad aparente $\left(d_{a}, \mathrm{gcm}^{-3}\right)$. Las muestras de suelo contenidas en anillos $\left(17.9 \mathrm{~cm}^{3}\right) \mathrm{se}$ colocaron en ollas de presión (Soil Moisture, modelos CAT 1500 y CAT 1600) para medir la retención de humedad gravimétrica a 0.033 $\mathrm{MPa}$ (CC, capacidad de campo) y $1.5 \mathrm{MPa}$ (PMP, punto de marchitez permanente). La diferencia entre CC y PMP se consideró como el agua disponible. La textura se determinó según la distribución porcentual (masa/masa) de partículas de arena $(2.00-0.05 \mathrm{~mm}$ diámetro), limo $(0.05-0.002 \mathrm{~mm})$ y arcilla $(<0.002$ $\mathrm{mm})$ mediante el método de Bouyoucos modificado (Bouyoucos 1951). Se determinó la densidad real o densidad de partículas minerales $\left(d_{p}, \mathrm{~g} \mathrm{~cm}^{-3}\right)$. El espacio poroso $(\mathrm{EP}$, en $\% \mathrm{~m} / \mathrm{m})$ de cada horizonte se calculó como $\mathrm{EP}=\left[1-\left(d_{a} /\right.\right.$ $\left.\left.d_{p}\right)\right]^{*} 100$. Finalmente, se calculó el $\%$ de microporos (diámetro $<0.2 \mu \mathrm{m}$ ) según la relación PMP x $d_{a}$; el \% de mesoporos (diámetro 0.2$10 \mu \mathrm{m})$ según la relación $\left(\mathrm{CC} \times d_{a}\right)$ - (PMP x $d_{a}$ ) y el $\%$ de macroporos (diámetro $\geq 10 \mu \mathrm{m}$ ) por diferencia, según la relación Macroporos = EP-Microporos-Mesoporos, para todos los horizontes.

Análisis estadísticos: Se realizaron análisis de estadística descriptiva, correlaciones de momento de Pearson (r) y regresiones de ajuste lineal $\left(r^{2}\right)$ con varios modelos (Zar 1999) para establecer tendencias a lo largo de la cronosecuencia sucesional. Los análisis se realizaron con Statistica (v. 5.5, Statsoft 2000). Debido a la variabilidad natural de las características físicas y químicas de los suelos, se realizaron análisis de correlación únicamente en suelos con contacto lítico (presencia de roca madre) a menos de $50 \mathrm{~cm}$ de profundidad, clasificados como Entisoles y Mollisoles, presentes en bosques con edades crecientes.

\section{RESULTADOS}

Subgrupos de suelos dominantes: Se observó alta variación edáfica en áreas $<1$ ha de bosques secundarios y en áreas $<0.5$ ha de bosques maduros (>100 años). Los sitios estudiados presentaron al menos dos subgrupos de suelos (Cuadro 1); en un sitio se identificaron tres subgrupos (Cuadro 1, sitios >100 años, e-fg). Los órdenes de suelo más comunes fueron los Entisoles y los Vertisoles, presentes en 44 y 27 de las 130 parcelas establecidas, respectivamente. Los demás órdenes identificados fueron Mollisoles (21 parcelas), Alfisoles (14 parcelas), Inceptisoles (13 parcelas) y Ultisoles (11 parcelas).

Características químicas y físicas de los suelos: La mayoría de los suelos presentaron bajo contenido de ceniza volcánica, con excepción de los suelos 20-a y 40-b (Cuadro 1). Los Entisoles y los Vertisoles estuvieron asociados a sitios planos ( $<3 \%$ pendiente $)$, mal drenados 
CUADRO 1

Suelos dominantes en ocho bosques tropicales secos de edades crecientes en Santa Rosa, Costa Rica

TABLE 1

Dominant soils in eight tropical dry forests of increasing age in Santa Rosa, Costa Rica

\begin{tabular}{|c|c|c|c|c|}
\hline $\begin{array}{l}\text { Edad * } \\
(\text { años })\end{array}$ & $\mathrm{n}$ & Clasificación del Suelo & $\begin{array}{c}\text { Pendiente } \\
(\%)\end{array}$ & Descripción del paisaje \\
\hline $10-\mathrm{a}$ & 12 & Vertisol, Ustic "Lithic" Epiaquerts & 1 & \multirow{6}{*}{$\begin{array}{l}\text { Relieve plano, pendientes }<3 \% \text {. Suelos poco } \\
\text { profundos, desarrollados sobre afloramientos de } \\
\text { ignimbritas consolidadas; se inundan durante } \\
\text { la estación lluviosa y se secan totalmente entre } \\
\text { diciembre-abril y en julio-agosto; condiciones } \\
\text { ideales para la formación de Entisoles y Vertisoles. }\end{array}$} \\
\hline $10-\mathrm{b}$ & 8 & Entisol, Typic "Lithic” Epiaquents & 1 & \\
\hline $15-\mathrm{a}$ & 13 & Entisol, Lithic Ustorthents & 1 & \\
\hline $15-b$ & 7 & Entisol, Typic "Vertic" Epiaquents & 1 & \\
\hline $20-\mathrm{a}$ & 12 & Entisol, Lithic "Andic" Ustorthents & 1 & \\
\hline $20-\mathrm{b}$ & 8 & Vertisol, Ustic Epiaquerts & 1 & \\
\hline $40-\mathrm{a}$ & 11 & Mollisol, Entic Haplustolls & 32 & \multirow{8}{*}{$\begin{array}{l}\text { Relieve predominantemente ondulado ( } 3-32 \% \\
\text { pendiente). Afloramientos de ignimbritas } \\
\text { consolidadas dominan las colinas. Suelos } \\
\text { bien drenados y de buenas características } \\
\text { físicoquímicas. Los suelos de los micro-valles } \\
\text { son más profundos y muestran mayor contenido y } \\
\text { acumulación de nutrimentos. }\end{array}$} \\
\hline $40-\mathrm{b}$ & 9 & Inceptisol, Andic Dystrustepts & 9 & \\
\hline $60-\mathrm{a}$ & 11 & Ultisol, Typic Haplustults & 20 & \\
\hline $60-b$ & 9 & Entisol, Typic Ustorthents & 3 & \\
\hline$>100-\mathrm{a}$ & 6 & Mollisol, Lithic Haplustolls & 3 & \\
\hline$>100-b$ & 4 & Mollisol, Entic Haplustolls & 15 & \\
\hline$>100-c$ & 6 & Alfisol, Ultic Haplustalfs & 6 & \\
\hline$>100-d$ & 4 & Inceptisol, Dystric Haplustepts & 32 & \\
\hline$>100-\mathrm{e}$ & 4 & Alfisol, Inceptic Haplustalfs & 3 & \multirow{3}{*}{$\begin{array}{l}\text { Relieve ligeramente ondulado (3-22\% pendiente). } \\
\text { Suelos asociados a dos terrazas aluviales }(\mathrm{e}, \mathrm{f}) \mathrm{y} \\
\text { un afloramiento rocoso }(\mathrm{g}) \text {, bien drenados y con } \\
\text { presencia de horizontes B argílicos (ricos en arcilla). }\end{array}$} \\
\hline$>100-f$ & 4 & Alfisol, Ultic Haplustalfs & 4 & \\
\hline$>100-\mathrm{g}$ & 2 & Entisol, Lithic Ustorthents & 22 & \\
\hline
\end{tabular}

*: a-g: pedones de suelos descritos, al menos dos en cada edad o bosque. $\mathbf{n}$ : número de parcelas de $500 \mathrm{~m}^{2}$ establecidas en cada pedón. Elementos entre comillas (e.g. "Lithic") no son parte de la taxonomía; son adiciones para explicar mejor las propiedades de los suelos.

La clasificación se realizó según el Soil Survey Staff (2007). Soil taxonomy classification was done following Soil Survey Staff (2007) criteria.

durante la estación lluviosa, con tendencia a acumular nutrimentos, poca profundidad, y presencia de ignimbritas o contactos líticos a poca profundidad (Cuadro 1; suelos 10-b, 15-a, 15-b, 20-a y 60-b).

Dentro de los Entisoles, el grupo de suelos más común fue el Ustorthents, presente en cuatro sitios, con escasa deposición de cenizas volcánicas y profundidades de la roca entre 22-60 $\mathrm{cm}$ (Cuadros 2 y 3 ). Estos suelos de color negro y grisáceo fueron clasificados como Vertisoles del subgrupo Ustic Epiaquerts (10-a y 20-b), expandibles durante la estación lluviosa y agrietados en la estación seca, con superficies de deslizamiento, contenidos de arcilla $>60 \%$ y CIC de aproximadamente $40 \mathrm{cmol}(+) \mathrm{kg}^{-1}$ en los horizontes B (Figs. 1 y 2). Los Entisoles y los Vertisoles dominaron las áreas planas encontradas en los bosques de 10 y 20 años, donde el relieve no facilitó la meteorización.

Los Mollisoles se encontraron en sitios con ignimbritas menos consolidadas o de grano suelto, relieves ondulados (3-32\% de pendiente), drenaje libre y colores oscuros, resultado de la adición continua de materia orgánica en los horizontes superficiales. Estos suelos mostraron alta saturación de bases $(>50 \%$ de la CIC, Fig. 1), texturas franco arenosas a franco arcillosas, conductividad hidráulica $k_{h}$ de moderada $\left(2-6 \mathrm{cmh}^{-1}\right)$ a muy alta $\left(>25 \mathrm{~cm} \mathrm{~h}^{-1}\right)$, 
CUADRO 2

Propiedades fisicas de los suelos clasificados como Entisoles, Vertisoles e Inceptisoles en BTS

de edades crecientes en Santa Rosa, Costa Rica

TABLE 2

Physical properties of soils classified as Entisols, Vertisols and Inceptisols in TDF of increasing age in Santa Rosa, Costa Rica

\begin{tabular}{|c|c|c|c|c|c|c|}
\hline $\begin{array}{l}\text { Edad } \\
\text { (años) }\end{array}$ & $\mathrm{H}$ & $\begin{array}{l}\text { Profundidad } \\
\text { (cm) }\end{array}$ & $\begin{array}{c}\mathrm{k}_{\mathrm{h}} \\
\left(\mathrm{cmh}^{-1}\right)\end{array}$ & $\begin{array}{c}\mathrm{d}_{\mathrm{a}} \\
\left(\mathrm{gcm}^{-3}\right)\end{array}$ & $\begin{array}{c}\mathrm{d}_{\mathrm{p}} \\
\left(\mathrm{gcm}^{-3}\right)\end{array}$ & $\begin{array}{l}\text { EP } \\
(\%)\end{array}$ \\
\hline \multicolumn{7}{|c|}{ Entisoles y Vertisoles } \\
\hline \multirow[t]{2}{*}{$10-\mathrm{a}$} & A & $0-17$ & 2.25 & 1.25 & 2.47 & 49 \\
\hline & Bss & $17-50^{*}$ & 0.75 & 1.61 & 2.35 & 32 \\
\hline $10-b$ & A & $0-22 *$ & 3.93 & 1.34 & 2.33 & 43 \\
\hline \multirow[t]{3}{*}{$15-\mathrm{a}$} & A & $0-13$ & 1.34 & 1.03 & 2.76 & 63 \\
\hline & $\mathrm{A} 2$ & $13-25$ & 2.27 & 1.21 & 2.62 & 54 \\
\hline & $\mathrm{Bw}$ & $25-38^{*}$ & 0.57 & 1.20 & 2.26 & 47 \\
\hline \multirow[t]{3}{*}{$15-\mathrm{b}$} & A & $0-18$ & 0.31 & 1.12 & 2.17 & 48 \\
\hline & $\mathrm{AC}$ & $18-40$ & $<0.10$ & 1.48 & 2.42 & 39 \\
\hline & $\mathrm{Cg}$ & $40-62 *$ & $<0.10$ & 1.42 & 2.55 & 44 \\
\hline \multirow[t]{2}{*}{$20-\mathrm{a}$} & A & $0-12$ & 7.50 & 0.99 & 2.38 & 58 \\
\hline & $\mathrm{A} 2$ & $12-22 *$ & 6.05 & 0.95 & 2.45 & 61 \\
\hline \multirow[t]{2}{*}{$20-b$} & A & $0-20$ & 1.70 & 1.18 & 2.33 & 49 \\
\hline & Bss & $20-64 *$ & 2.27 & 1.39 & 2.34 & 41 \\
\hline \multirow[t]{4}{*}{$60-\mathrm{b}$} & A & $0-15$ & 18.00 & 0.96 & 2.34 & 59 \\
\hline & $\mathrm{A} 2$ & $15-33$ & 8.55 & 0.89 & 2.18 & 59 \\
\hline & $\mathrm{C}$ & $33-47$ & 12.00 & 0.90 & 2.48 & 64 \\
\hline & $\mathrm{C} 2$ & $47-72 *$ & $<0.10$ & 1.30 & 2.39 & 46 \\
\hline$>100-\mathrm{g}$ & A & $0-22$ & 22.00 & 1.00 & 2.46 & 60 \\
\hline \multicolumn{7}{|c|}{ Inceptisoles } \\
\hline \multirow[t]{6}{*}{$40-b$} & A & $0-20$ & 9.77 & 1.17 & 2.62 & 55 \\
\hline & $\mathrm{A} 2$ & $20-44$ & 5.56 & 1.07 & 2.64 & 60 \\
\hline & A3 & $44-70$ & 5.45 & 0.86 & 2.74 & 69 \\
\hline & A4 & $70-98$ & 5.49 & 0.90 & 2.69 & 67 \\
\hline & $\mathrm{C}$ & $98-108$ & 5.38 & 0.68 & 2.59 & 74 \\
\hline & $\mathrm{Ab}$ & $108-162+$ & 1.55 & 1.17 & 2.55 & 54 \\
\hline \multirow[t]{7}{*}{$>100-d$} & A & $0-14$ & 4.26 & 1.18 & 2.21 & 47 \\
\hline & $\mathrm{Bw}$ & $14-28$ & 2.49 & 1.16 & 2.26 & 49 \\
\hline & Bw2 & $28-53$ & 2.27 & 1.06 & 2.29 & 54 \\
\hline & $\mathrm{BC}$ & $53-77$ & $<0.10$ & 1.20 & 2.27 & 47 \\
\hline & $\mathrm{C}$ & $77-90$ & 0.91 & 1.01 & 2.30 & 56 \\
\hline & $\mathrm{C} 2$ & $90-103$ & 0.24 & 1.07 & 2.33 & 54 \\
\hline & $\mathrm{Cr}$ & $103-174 *$ & 1.30 & 1.05 & 2.23 & 53 \\
\hline
\end{tabular}

Simbología. Edad: Ver Cuadro 1. H: Letras mayúsculas indican los horizontes del suelo. Letras minúsculas indican: $\boldsymbol{b}$ : horizonte enterrado, $\boldsymbol{g}$ : colores grisáceos por mal drenaje, $\boldsymbol{r}$ : roca suave o meteorizada, ss: presencia de superficies de deslizamiento, $\boldsymbol{t}$ : acumulación de arcilla, $\boldsymbol{w}$ : desarrollo de color o estructura (Schoeneberger et al. 2002). $\mathbf{k}_{\mathbf{h}}$ : conductividad hidráulica. $\mathbf{d}_{\mathrm{a}}$ : densidad aparente. $\mathbf{d}_{\mathrm{p}}$ : densidad de partículas. EP: Espacio poroso. +: posible continuidad del horizonte. *: rocas consolidadas a la máxima profundidad examinada. 
CUADRO 3

Propiedades físicas de los suelos clasificados como Mollisoles, Alfisoles y Ultisoles en BTS de edades crecientes en Santa Rosa, Costa Rica

TABLE 3

Physical properties of soils classified as Mollisols, Alfisols and Ultisols in TDF of increasing age in Santa Rosa, Costa Rica

\begin{tabular}{|c|c|c|c|c|c|c|}
\hline $\begin{array}{l}\text { Edad } \\
\text { (años) }\end{array}$ & $\mathrm{H}$ & $\begin{array}{l}\text { Profundidad } \\
\quad(\mathrm{cm})\end{array}$ & $\begin{array}{c}\mathrm{k}_{\mathrm{h}} \\
\left(\mathrm{cmh}^{-1}\right)\end{array}$ & $\begin{array}{c}\mathrm{d}_{\mathrm{a}} \\
\left(\mathrm{gcm}^{-3}\right)\end{array}$ & $\begin{array}{c}\mathrm{d}_{\mathrm{p}} \\
\left(\mathrm{gcm}^{-3}\right)\end{array}$ & $\begin{array}{l}\text { EP } \\
(\%)\end{array}$ \\
\hline \multicolumn{7}{|c|}{ Mollisoles } \\
\hline \multirow[t]{5}{*}{$40-\mathrm{a}$} & A & $0-6$ & 6.60 & 0.98 & 2.54 & 61 \\
\hline & $\mathrm{A} 2$ & $6-21$ & 24.43 & 1.10 & 2.65 & 59 \\
\hline & $\mathrm{AC}$ & $21-35$ & 8.03 & 1.21 & 2.61 & 54 \\
\hline & $\mathrm{C}$ & $35-60$ & 4.35 & 1.29 & 2.61 & 51 \\
\hline & $\mathrm{Cr}$ & $60-170+$ & 4.68 & 1.08 & 2.44 & 56 \\
\hline \multirow[t]{3}{*}{$>100-\mathrm{a}$} & $\mathrm{A}$ & $0-13$ & 12.47 & 1.09 & 2.45 & 56 \\
\hline & $\mathrm{A} 2$ & $13-25$ & 33.98 & 1.01 & 2.54 & 60 \\
\hline & $\mathrm{BC}$ & $24-40^{*}$ & 18.85 & 1.10 & 2.51 & 56 \\
\hline \multirow[t]{6}{*}{$>100-b$} & A & $0-42$ & 11.71 & 0.95 & 2.36 & 60 \\
\hline & $\mathrm{Ab}$ & $42-76$ & 8.97 & 0.93 & 2.43 & 62 \\
\hline & A3 & $76-112$ & 5.14 & 1.05 & 2.52 & 58 \\
\hline & $\mathrm{AC}$ & $112-138$ & 11.21 & 1.01 & 2.62 & 62 \\
\hline & $\mathrm{C}$ & $138-158$ & 16.45 & 0.99 & 2.69 & 63 \\
\hline & $\mathrm{Ab} 2$ & $159-192+$ & 6.20 & 1.03 & 2.49 & 59 \\
\hline \multicolumn{7}{|c|}{ Alfisoles y Ultisoles } \\
\hline \multirow[t]{5}{*}{$60-\mathrm{a}$} & A & $0-20$ & $n d$ & 0.89 & 2.36 & 62 \\
\hline & $\mathrm{AB}$ & $20-30$ & nd & 1.29 & 2.41 & 47 \\
\hline & $\mathrm{Bt}$ & $30-78$ & $n d$ & 1.27 & 2.38 & 47 \\
\hline & $\mathrm{BC}$ & $78-100$ & $n d$ & 1.22 & 2.40 & 49 \\
\hline & $\mathrm{C}$ & $100-140+$ & $n d$ & 1.20 & 2.29 & 48 \\
\hline \multirow[t]{4}{*}{$>100-\mathrm{c}$} & A & $0-19$ & 2.10 & 1.18 & 2.36 & 50 \\
\hline & $\mathrm{A} 2$ & $19-45$ & 0.01 & 1.46 & 2.32 & 37 \\
\hline & $\mathrm{Bt}$ & $45-58$ & 0.01 & 1.54 & 2.35 & 35 \\
\hline & Bt2 & $58-116+$ & 0.01 & 1.53 & 2.32 & 34 \\
\hline \multirow[t]{5}{*}{$>100$-e } & A & $0-10$ & 41.41 & 0.93 & 2.42 & 62 \\
\hline & $\mathrm{A} 2$ & $10-23$ & 7.44 & 1.13 & 2.46 & 54 \\
\hline & $\mathrm{C}$ & $23-51$ & 22.66 & 0.89 & 2.46 & 64 \\
\hline & $\mathrm{Ab}$ & $51-74$ & 6.89 & 1.17 & 2.51 & 53 \\
\hline & $\mathrm{Bt} 1$ & $74-105+$ & 0.06 & 1.51 & 2.53 & 40 \\
\hline \multirow[t]{4}{*}{$>100-f$} & A & $0-6$ & 17.76 & 0.99 & 2.42 & 59 \\
\hline & Bt1 & $6-24$ & 2.99 & 1.13 & 2.41 & 53 \\
\hline & $\mathrm{Ab}$ & $24-57$ & 1.29 & 1.37 & 2.46 & 44 \\
\hline & $2 \mathrm{Bt} 2$ & $57-100+$ & 0.11 & 1.50 & 2.56 & 41 \\
\hline
\end{tabular}

Simbología: ver Cuadro 2. 


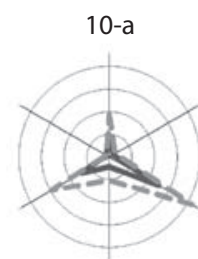

20-a

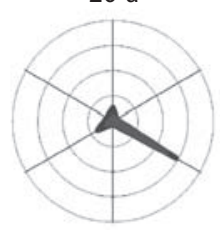

$>100-a$

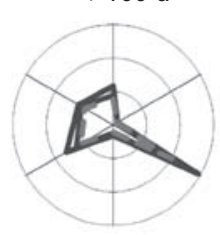

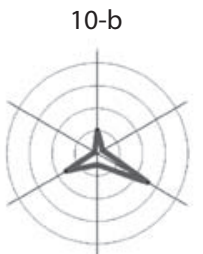

20-b

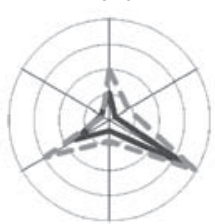

$>100-b$

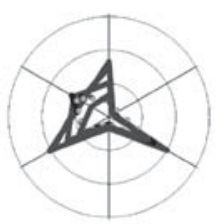

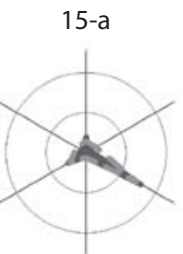

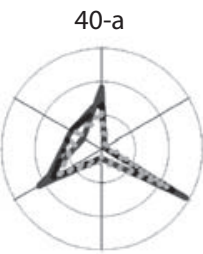

$>100-\mathrm{C}$

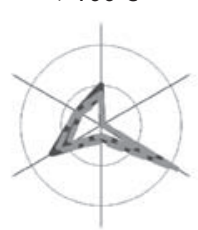

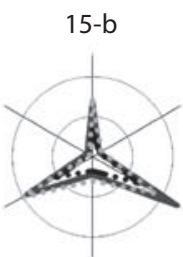

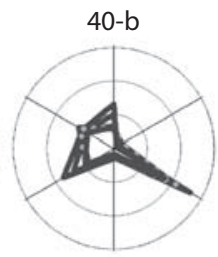

$>100-d$

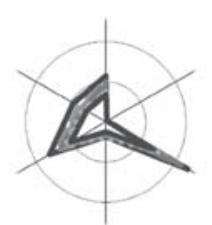

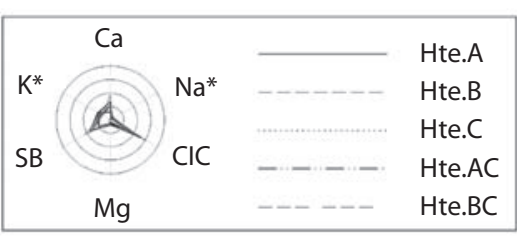
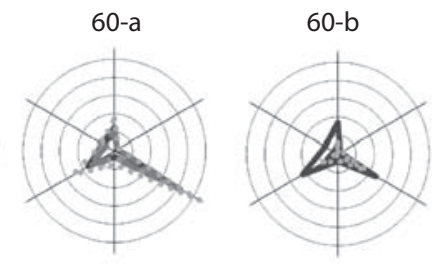

$>100-$ e

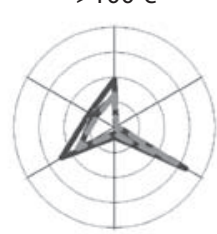

$>100-f \&>100 g$

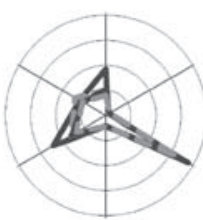

Fig. 1. Contenido de bases disponibles $\left(\mathrm{cmol}(+) \mathrm{kg}^{-1}\right)$ en horizontes (A, B, C, AC y BC) de 17 suelos a lo largo de una cronosecuencia de regeneración del BTS en Santa Rosa, Costa Rica. Los ejes representan seis variables: Ca. Mg, SB (suma de bases), CIC (capacidad de intercambio catiónico), Na y K. *Na y K en mmol(+) $\mathrm{kg}^{-1}$. Cada anillo representa incrementos de 10 unidades.

Fig. 1. Available cations content $\left(\mathrm{cmol}(+) \mathrm{kg}^{-1}\right)$ in horizons $(\mathrm{A}, \mathrm{B}, \mathrm{C}, \mathrm{AC}$ y BC) of 17 soils in TDF of increasing age at Santa Rosa, Costa Rica. Six variables represented by axes: $\mathrm{Ca}, \mathrm{Mg}, \mathrm{SB}$ (sum of cations), CIC (cation exchange capacity), Na and $\mathrm{K} .{ }^{*} \mathrm{Na}$ and $\mathrm{K}$ expressed in $\mathrm{mmol}(+) \mathrm{kg}^{-1}$. Each ring represents 10 units of increment.

porosidad adecuada (EP $>50 \%$ ) y profundidad efectiva $>1.5 \mathrm{~m}$ en dos perfiles (Cuadro 3 ).

Los Alfisoles y Ultisoles presentaron un aumento en el contenido de arcilla con la profundidad, especialmente en los horizontes argílicos del subsuelo (B, 38-61\% de arcilla, Fig. 2), películas conspicuas de arcillas (argicutanes) y colores pardos rojizos (Cuadro 3). El grangrupo más común fue Haplustalfs, presente en dos BTS maduros (>100 años, c-e-f). Algunos Alfisoles se clasificaron como intergradaciones con Ultisoles (Subgrupos Ultic, pedón 100-c y 100-f), pues mostraron menor contenido de nutrimentos, característico de ese orden de suelo. Los Inceptisoles se presentaron en dos sitios (clases 40-b y >100-d) y mostraron perfiles de desarrollo intermedio entre los Entisoles y los Mollisoles. El suelo Dystric Haplustepts ( $>100$ d) se consideró como un suelo en desarrollo intermedio entre los Entisoles y los Ultisoles.

En general, los suelos clasificados como Vertisoles, Alfisoles y Ultisoles presentaron texturas franco-arenosas a franco-arcillosas en los horizontes A superficiales (8-41\% arcilla) y texturas franco-arcillosas a arcillosas en los horizontes del subsuelo (28-61\% arcilla). Estos suelos mostraron $d_{a}=1.15-1.60 \mathrm{gcm}^{-3}$, agua disponible entre $2-8 \%$, y $k_{h}$ muy baja a moderada $\left(<0.13-6.30 \mathrm{cmh}^{-1}\right)$ en los horizontes del subsuelo. Los suelos clasificados como Entisoles, Inceptisoles y Mollisoles presentaron texturas francas a franco-arenosas en todo el perfil (48$82 \%$ arena), las menores $d_{a}\left(0.80-1.15 \mathrm{gcm}^{-3}\right) \mathrm{y}$ 


\section{Bosques en regeneración}

Simbología

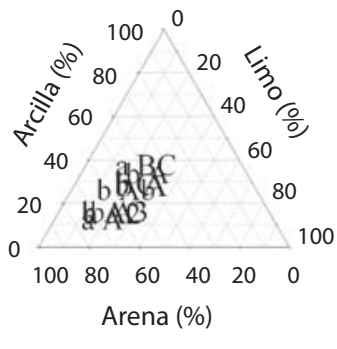

20 años

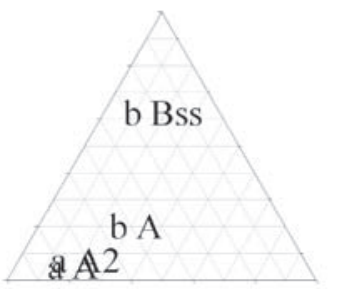

Mollisoles*

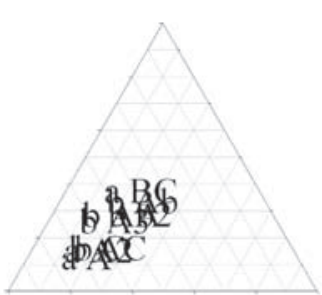

10 años

a Bss

baA

40 años

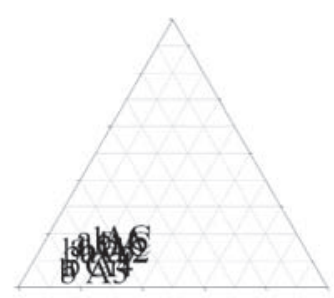

Bosques maduros

Alfisoles*

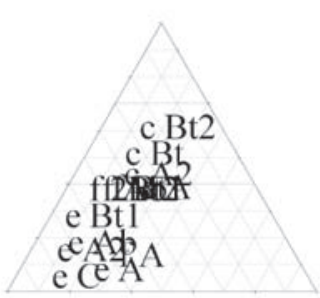

15 años

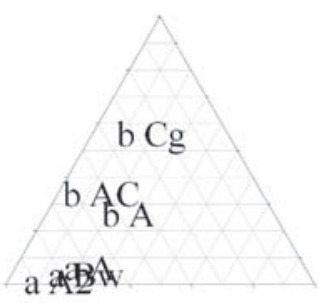

60 años

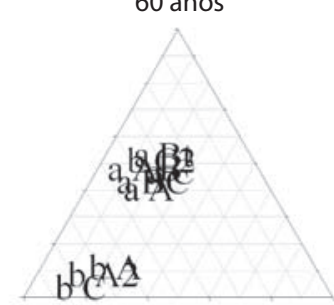

Entisoles e Inceptisoles*

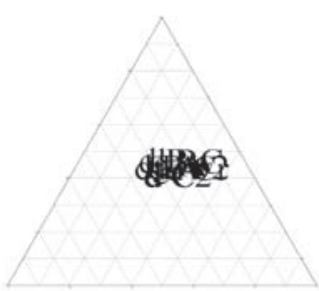

Fig. 2. Textura del suelo según los porcentajes de partículas minerales finas $(<2 \mathrm{~mm})$ en muestras de los horizontes en 17 suelos descritos en BTS de edades crecientes en Santa Rosa, Costa Rica. En cada edad se muestra el pedón (según el Cuadro 1) y el horizonte descrito (p.e.: A, Bt, C). *En los bosques maduros se muestran datos separados por orden de suelo.

Fig. 2. Soil texture according to fine particles percentages $(<2 \mathrm{~mm})$ of samples from all horizons in 17 soils in TDF of increasing age in Santa Rosa, Costa Rica. For every age soil pedons (Table 1) and horizons (e.g.: A, Bt, C) are described. *Data arranged by soil orders in mature forests.

las mayores $k_{h}\left(6.3-33.0 \mathrm{cmh}^{-1}\right)$. Estos valores de $d_{p}$ indicaron similitud en la constitución de los materiales parentales que dieron origen a estas clases de suelos (Cuadros 2 y 3 ).

Cambios en el contenido de cationes con la regeneración boscosa: El efecto de la regeneración boscosa fue evidente sobre el contenido de $\mathrm{Ca}, \mathrm{Mg}, \mathrm{K}$, Na y CIC extraídos en acetato de amonio (Fig. 3). Durante los primeros 20 años de regeneración en Entisoles se notó una disminución considerable de las bases de intercambio. Los cationes intercambiables se incrementaron a partir de sesenta años, y fueron similares a los medidos en los estados sucesionales más avanzados. Estos resultados fueron corroborados mediante la utilización de otras soluciones extractoras ( $\mathrm{KCl}$, Olsen modificada; 


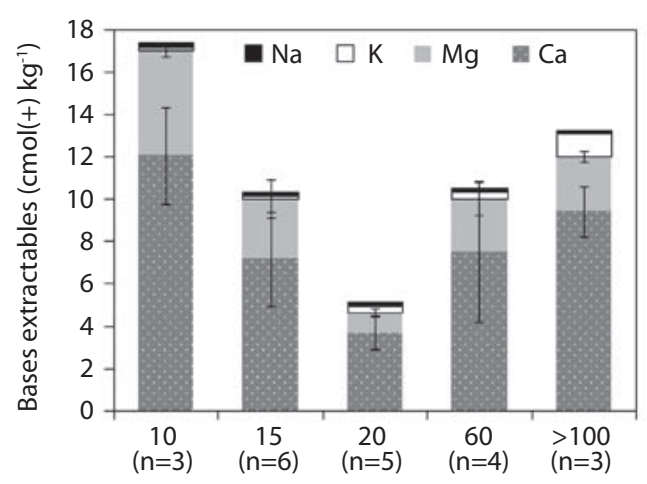

Edad del bosque (años)

Fig. 3. Contenido de $\mathrm{Ca}, \mathrm{Mg}, \mathrm{K}$ y $\mathrm{Na}$ del suelo a lo largo de una cronosecuencia de regeneración del BTS en Santa Rosa, Costa Rica. Datos para cuatro bosques secundarios y un bosque maduro ( $>100$ años), en suelos con contacto lítico $<50 \mathrm{~cm}$. Se muestra valor \pm error estándar para cada variable.

Fig. 3. Soil content of $\mathrm{Ca}, \mathrm{Mg}, \mathrm{K}$ and $\mathrm{Na}$ during TDF regeneration at Santa Rosa, Costa Rica. Data \pm standard error for four secondary and one mature TDF ( $>100$ años), in soils with lithic contact $<50 \mathrm{~cm}$ deep.

datos no presentados). Las correlaciones entre el contenido de cationes extraídos con acetato de amonio y la edad del bosque no fueron significativas. El potasio disponible fue la única excepción, pues su nivel aumentó conforme se incrementó la regeneración boscosa $(r=0.77$, $\mathrm{p}<0.001)$. Los demás nutrimentos no presentaron variaciones importantes a lo largo de la sucesión, aunque se observaron correlaciones positivas y significativas entre ellos $(0.66 \leq \mathrm{r} \leq$ 0.99). Los elementos menores tampoco variaron significativamente con la sucesión (datos no mostrados). No se observaron cambios en el contenido de materia orgánica en los 30 $\mathrm{cm}$ superficiales del suelo $(\mathrm{r}=0.03, \mathrm{p}=0.89)$ a lo largo de la sucesión, aunque la materia orgánica disminuyó significativamente con la profundidad $(r=-0.81, \mathrm{p}<0.001$, Fig. 4$)$.

Cambios en las propiedades físicas con la regeneración boscosa: Las propiedades físicas de los Entisoles, el orden de suelos más abundante, variaron significativamente conforme progresó la sucesión. El agua disponible $(\mathrm{r}=-0.54, \mathrm{p}=0.03)$ y la abundancia de mesopo$\operatorname{ros}(\mathrm{r}=-0.72, \mathrm{p}=0.002)$ disminuyeron significativamente durante la regeneración (Fig. 5), porque $k_{h}(\mathrm{r}=0.79, \mathrm{p}<0.001)$ y los macroporos $(\mathrm{r}=0.59, \mathrm{p}=0.016)$ se incrementaron con la sucesión (Fig. 5). La $k_{h}$ aumentó y el agua disponible disminuyó $(\mathrm{r}=0.94, \mathrm{p}<0.001)$ con la abundancia de macroporos ( $\mathrm{r}=0.65 ; \mathrm{p}<0.006$ ), pero estuvieron relacionadas positivamente con la abundancia de mesoporos.

\section{DISCUSIÓN}

Nuestros resultados revelan alta diversidad espacial a escalas pequeñas entre los suelos del BTS de Santa Rosa, Costa Rica. Se describieron clases pertenecientes a seis órdenes de la taxonomía de los suelos, resultado de complejas interacciones entre los materiales parentales ignimbríticos, la estacionalidad climática, la erosión, la topografía, los organismos, y la intervención humana. Winters (1997) obtuvo resultados similares y reconoció siete divisiones o clases de suelos. Estas variaciones han sido implicadas en importantes procesos durante la regeneración boscosa, como la germinación de las semillas y el establecimiento de las plántulas (Gerhardt 1993), y sus implicaciones sobre la regeneración boscosa asociada a estos suelos son examinadas en una publicación adjunta.

La existencia de clases típicas de suelos viejos, profundos y meteorizados como los Alfisoles y los Ultisoles en Santa Rosa es consistente con la edad de los flujos piroclásticos que dieron origen a la MISR. Estos suelos presentaron acumulación de arcillas en los horizontes B (argílicos), bloqueando el espacio poroso y formando argicutanes sobre la superficie de los agregados y los poros. Estos suelos mostraron mayor madurez, drenaje bueno a moderado, y acumularon bases de intercambio (Buol et al. 1989).

Los Mollisoles, que en zonas templadas se originan por la acumulación de materia orgánica en las praderas, también se desarrollaron en 


\section{Contenido de Materia Orgánica (\%)}

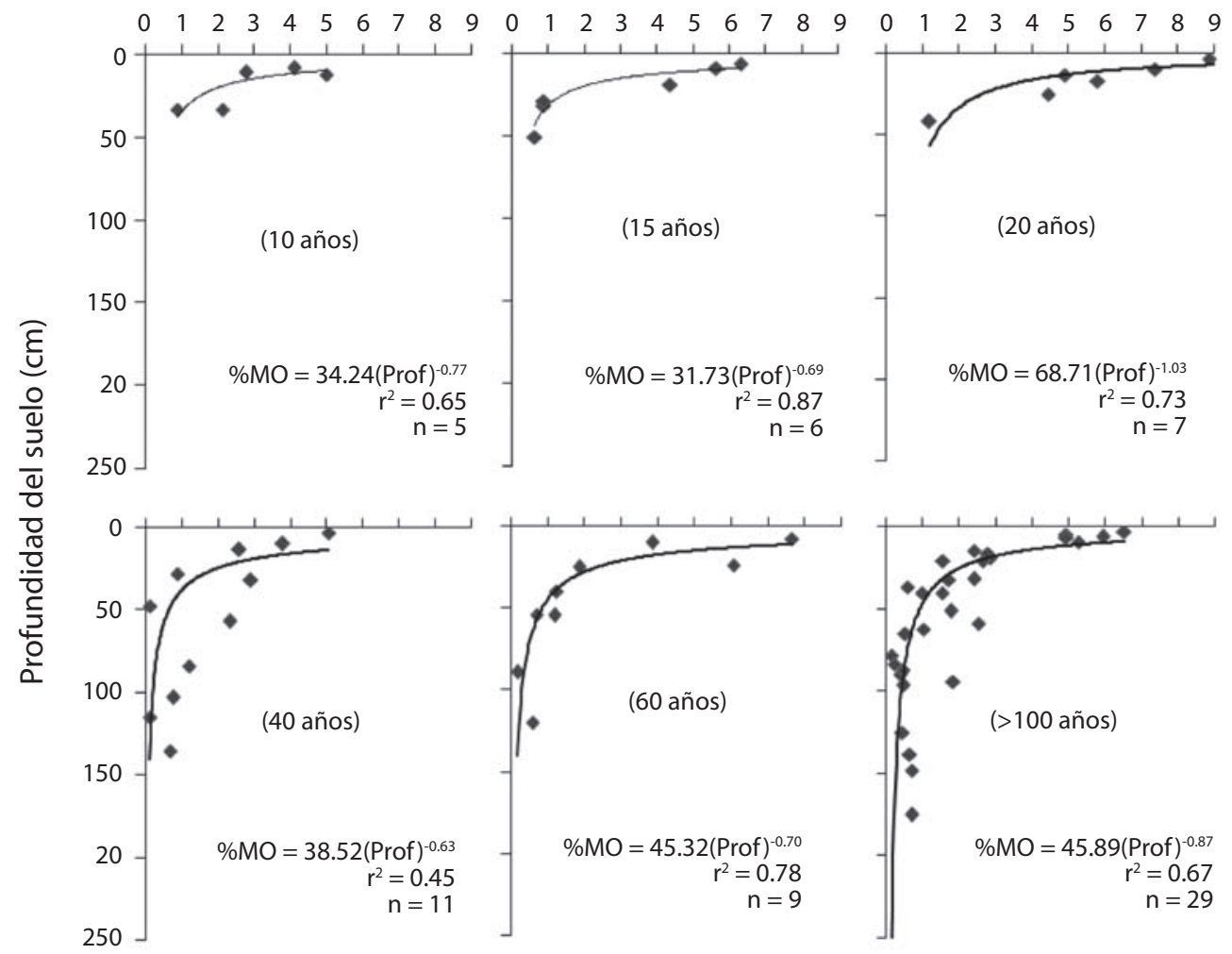

Fig. 4. Contenido de materia orgánica (\%) según la profundidad del suelo $(\mathrm{cm})$ a lo largo de una cronosecuencia de regeneración del BTS de Santa Rosa, Costa Rica. Para todos los horizontes de suelo descritos $(\mathrm{n}=67)$ la relación exponencial fue: Materia Orgánica $(\%)=42.18(\text { Prof })^{-0.80}, \mathrm{r}^{2}=0.65$.

Fig. 4. Soil organic matter (\% MO) according to successional age (years) and soil depth $(\mathrm{cm})$. For all horizons described (n $=67)$ the exponential relation was: Organic matter $(\%)=42.18(\text { Prof })^{-0.80}, r^{2}=0.65$.

Santa Rosa. Estos suelos fueron identificados en sitios de mayor edad (40 y >100 años), y su desarrollo pudo deberse al clima estacionalmente seco que propició la acumulación anual de hojarasca y material radicular, parcialmente descompuestos, en los horizontes superficiales (Jaramillo \& Sanford 1995). Además, el microclima generado durante la regeneración del dosel boscoso favoreció la pedoturbación constante (mezcla del suelo por acción de diversos organismos), que ha homogenizado, oscurecido y enriquecido el suelo con nutrimentos y materia orgánica.
La regeneración boscosa del BTS tuvo importantes efectos sobre algunas propiedades químicas y físicas de los suelos. La dinámica y abundante biomasa radical de los BTS (Cuevas 1995) probablemente jugó un papel central en los cambios observados en la porosidad y en la conductividad hidráulica, y del agua disponible para las plantas. Dentro de los Entisoles poco profundos se encontraron relaciones entre la edad del bosque y los niveles de $\mathrm{Ca}, \mathrm{Mg}, \mathrm{K}$ y $\mathrm{Na}$ intercambiables. Esto podría resultar en cambios en la diversidad de las especies a lo largo del proceso de regeneración, en vista de 

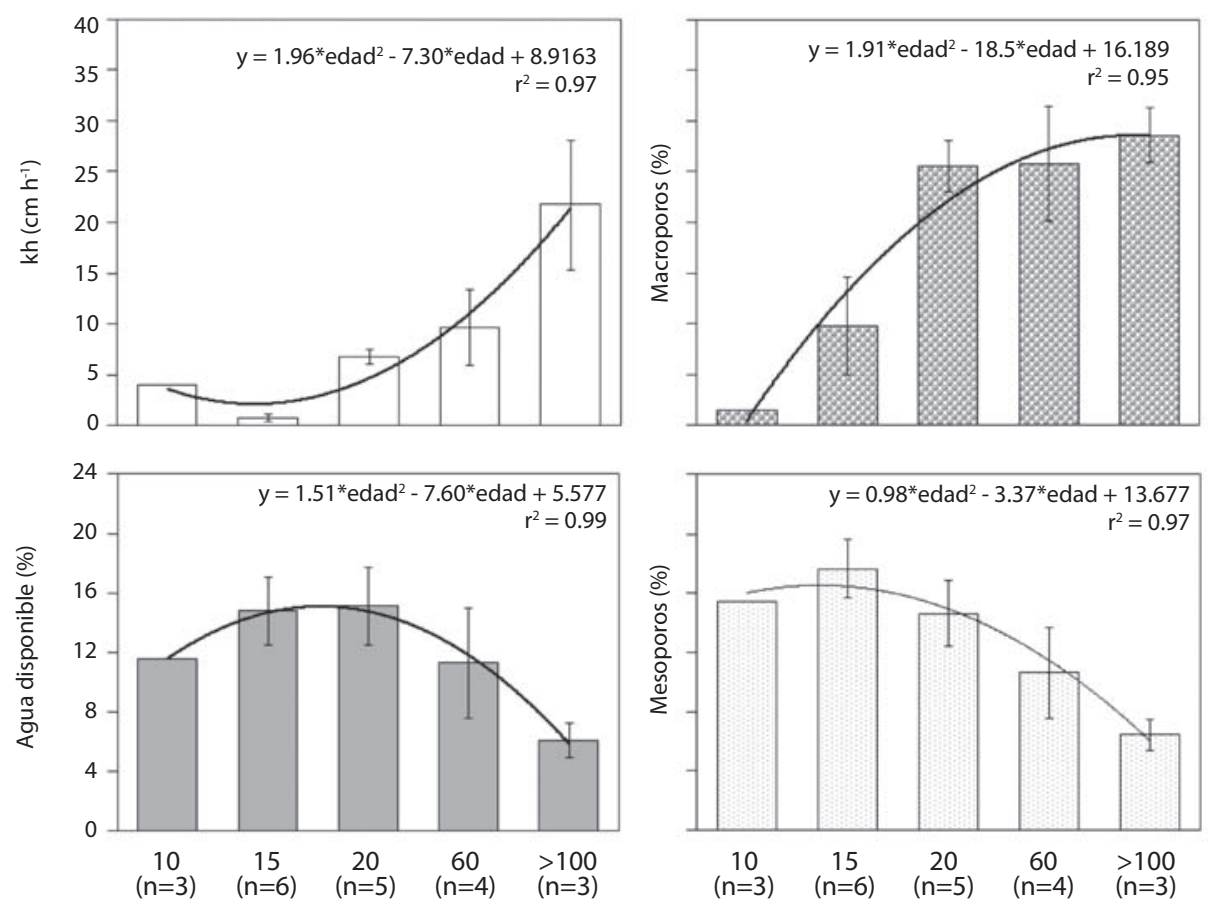

Edad del bosque (años)

Edad del bosque (años)

Fig. 5. Conductividad hidráulica (kh), macroporos (\%), agua disponible (\%) y mesoporos (\%) del suelo, a lo largo de una cronosecuencia de regeneración en cuatro BTS secundarios y uno maduro (BH) en Guanacaste, Costa Rica. Datos de suelos con contacto lítico $<50 \mathrm{~cm}$.

Fig. 5. Hydraulic conductivity (kh), macropores \%, soil available water (\%) and mesopores (\%), with increasing successional age in four secondary and one old growth TDF in Guanacaste, Costa Rica. Data for soils with lithic contact at depth $<50 \mathrm{~cm}$.

que especies con diferente fenología pueden preferir condiciones edáficas contrastantes (Huston 1980, Goldberg 1982). Aunque los cationes disponibles en los Entisoles disminuyeron en bosques de 10-20 años, esta reducción se desaceleró luego de 20 años. El contenido de bases de intercambio $(\mathrm{Ca}, \mathrm{Mg}$, K y CICE) aumentó hasta los 60 años, a partir de los cuales se registraron niveles similares a los encontrados en los suelos de BTS maduros (60 y >100 años) (Fig. 1) (Cuadros 2 y 3). En sitios sucesionales jóvenes, las quemas anuales de Hyparrhenia rufa y otras poáceas (Daubenmire 1972) pueden ser la causa de los aumentos generalizados en los contenidos de nutrimentos del suelo, principalmente de $\mathrm{Ca}$, Mg y K (Certini 2005). Luego del abandono de los pastizales, la erosión eólica e hídrica causaron la pérdida y lixiviación de bases, y la absorción de nutrimentos por parte de las especies pioneras redujeron los niveles de bases del suelo. El aumento subsecuente en el contenido de nutrimentos durante la sucesión pudo deberse a los pulsos de descomposición de hojarasca y raíces finas que adicionan materia orgánica y minerales al suelo (Cuevas 1995). La fijación biológica de nitrógeno atmosférico realizada por los miembros de Fabaceae, dominantes en estos bosques (Gentry 1995), la moderación del microclima por la acción 
del dosel boscoso (Maas 2005), y el reciclaje y la adición de nutrimentos por la escorrentía de las copas y los fustes, probablemente contribuyeron también en este efecto.

El contenido de materia orgánica del suelo (MO) no sufrió cambios durante la regeneración boscosa (Fig. 4), pero sí con la profundidad, lo que constituye un comportamiento normal de los procesos de regeneración arbórea del trópico (Jordan 1985). Aunque nuestras observaciones (Rocha et al., sin publicar) indican que la adición de hojarasca al suelo se incrementa con la regeneración boscosa, su reciclaje y la mayor actividad radical en los horizontes superficiales del suelo resultó en mayores contenidos de MO solo en esos horizontes (Fig. 4), y probablemente se reflejó en el mantenimiento de la fertilidad relativamente alta a lo largo de la crono-secuencia sucesional.

La alta relación biomasa radical: biomasa aérea típica de los BTS (0.08-0.45, incluso 1.01 ), considerablemente mayor que en bosques húmedos (Sanford y Cuevas 1996), probablemente jugó un papel importante en el aumento de la macroporosidad y la $\mathrm{k}_{\mathrm{h}}$ detectados en los Entisoles (Fig. 5). El clima estacional de Santa Rosa genera pulsos anuales de crecimiento y muerte de las raíces y de adición de materia orgánica, que contribuyen a la formación de macroporos en los horizontes superficiales del suelo (Cuevas 1995).

La regeneración del BTS propició el desarrollo de la biomasa radical (Raich 1980) e intensa pedoturbación, y favoreció la formación de macroporos en la matriz del suelo, mejorando la estructura y la aireación, aumentado la macroporosidad y la $\mathrm{k}_{\mathrm{h}}$, pero disminuyendo el contenido de agua disponible para las plantas. Esto podría modificar la severidad de la sequía experimentada por diferentes formas de vida en diferentes estados de sucesión (Hacke et al. 2000), así como las respuestas fenológicas de las plantas, que podrían experimentar déficit hídricos más severos y mayor competencia por los recursos del suelo en estados más avanzados de sucesión.

\section{AGRADECIMIENTOS}

Este trabajo fue financiado por el Programa de Ecología Terrestre de la Fundación Andrew W. Mellon. Agradecemos al personal del Parque Nacional Santa Rosa, especialmente a Róger Blanco y a María Marta Chavarría. Alfredo Alvarado y Ruperto Quesada hicieron valiosas sugerencias al trabajo de campo y al borrador del manuscrito.

\section{RESUMEN}

Se caracterizaron las propiedades químicas y físicas de los suelos a lo largo de una cronosecuencia de ocho fragmentos de bosque tropical estacionalmente seco (BTS) en Santa Rosa, Costa Rica, y se relacionaron con el estado sucesional de los sitios $(10,15,20,40,60$ y >100 años de regeneración). Se encontró una alta diversidad edáfica a escalas pequeñas (0.5-1 ha) y se describieron 17 clases dominantes de suelos, clasificados en seis órdenes. La mayoría de los suelos fueron clasificados como Entisoles y Vertisoles, pero los Mollisoles, Alfisoles, Inceptisoles y Ultisoles estuvieron representados también. Todos los suelos presentaron buenos índices de fertilidad que no restringieron la riqueza de las especies. La alta heterogeneidad edáfica pareció ser el resultado de la interacción entre el origen piroclástico de la meseta ignimbrítica de Santa Rosa, la composición litológica y la acidez de las rocas del material parental, la marcada estacionalidad de la lluvia, intensos procesos de erosión hídrica y eólica, variaciones topográficas locales, y la historia de intervención humana. Correlaciones efectuadas en los Entisoles ( $<60 \mathrm{~cm}$ profundidad) revelaron un agotamiento de los cationes (Ca, Mg, K, Na y CIC) durante los primeros veinte años de regeneración, que más tarde se recuperaron en los bosque maduros. El contenido de materia orgánica no varió significativamente con la regeneración. Se observaron incrementos sustanciales en los macroporos y en la conductividad hidráulica, probablemente resultado de mayor biomasa y actividad radical en sitios sucesionales más viejos. La abundancia de mesoporos y el agua disponible para las plantas disminuyeron con la regeneración del bosque. Los resultados indican que los cambios observados en el suelo son resultado de la presencia anual de fuego, la adición de materia orgánica y minerales al suelo conforme la regeneración progresó, las condiciones microclimáticas más benignas conforme el bosque se desarrolló, el creciente ciclaje de nutrimentos, y la predominancia de texturas franco-arenosas en los suelos examinados. Estos cambios en las propiedades del suelo con la sucesión pueden tener importantes consecuencias sobre la fisiología y la fenología de las diversas formas de vida vegetal observadas durante la regeneración de los bosques tropicales estacionalmente secos. 
Palabras clave: Parque Nacional Santa Rosa, suelos, propiedades químicas, propiedades físicas, bosque seco tropical estacional, pasturas, regeneración arbórea, sucesión.

\section{REFERENCIAS}

Alfaro, E.A., A. Alvarado \& A. Chaverri. 2001. Cambios edáficos asociados a tres etapas sucesionales de bosque tropical seco en Guanacaste, Costa Rica. Agron. Costarric. 25: 7-20.

Bazzaz, F.A. 1979. The physiological ecology of plant succession. Ann. Rev. Ecol. Syst. 10: 351-371.

Bazzaz, F.A. \& S.T.A. Pickett. 1980. Physiological ecology of tropical succession: a comparative review. Ann. Rev. Ecol. Syst. 11: 287-310.

Bouyoucos, G.J. 1951. A recalibration of the hydrometer method for making mechanical analyses of soils. Agron. J. 43: 434-438.

Buol, S.W., F.D. Hole \& R.J. McCraken. 1989. Soil Genesis and Classification. Iowa State University, Iowa, EEUU.

Certini, G. 2005. Effects of fire on properties of forest soils: a review. Oecologia 143: 1-10.

Chazdon, R.L. 2003. Tropical forest recovery: legacies of human impact and natural disturbances. Perspect. Plant. Ecol. 6: 51-71.

Chiesa, S. 1991. El flujo de pómez biotítico del Río Liberia (Guanacaste) Costa Rica, América Central. Rev. Geol. Amér. Centr. 13: 73-84.

Clark, D.B., M.W. Palmer \& D.A. Clark. 1999. Edaphic factors and the landscape-scale distributions of tropical rain forest trees. Ecology 80: 2662-2675.

Clark, D.B. 2002. Los factores edáficos y la distribución de las plantas, p. 193-221. In M.R. Guariguata \& G.H. Kattan (eds.). Ecología y conservación de bosques neotropicales. LUR, Cartago, Costa Rica.

Cuevas, E. 1995. Biology of the belowground system of tropical dry forest, p. 362-383. In S.H Bullock, H.A. Mooney \& E. Medina (eds.). Seasonally dry tropical forests. Cambridge University, Cambridge, Reino Unido.

Daubenmire, R. 1972. Ecology of Hyparrhenia rufa (Ness) in derived savanna in north-western Costa Rica. J. Appl. Ecol. 9: 11-23.

Ewel, J. 1980. Tropical succession: manifold routes to maturity. Biotropica 12: 2-7.
Fields, M. \& K.W. Perrott. 1966. The nature of allophane in soils. Part 3: Rapid field and laboratory tests for allophane. New Zeal. J. Sci. 9: 623-629.

Gentry, A.H. 1995. Diversity and floristic composition of neotropical dry forests, p. 146-194. In S.H Bullock, H.A. Mooney \& E. Medina (eds.). Seasonally dry tropical forests. Cambridge University, Cambridge, Reino Unido.

Gerhardt, K. 1993. Tree seedling development in abandoned pasture and secondary forest in former tropical dry forest in Guanacaste, Costa Rica. J. Veg. Sci. 4: 95-102

Gillot, P., S. Chiesa \& G.E. Alvarado. 1994. Chronostratigraphy of upper Miocene-Quaternary volcanism in Northern Costa Rica. Rev. Geol. Amér. Centr. 17: 45-53.

Goldberg, D.E. 1982. The distribution of evergreen and deciduous trees relative to soil type: an example from the Sierra Madre, México, and a general model. Ecology 63: 942-951.

Guariguata, M.R. \& R. Ostertag. 2001. Neotropical secondary forest succession: changes in structural and functional characteristics. Forest Ecol. Manag. 148: 185-206.

Hacke, U.G., J.S. Sperry, B.E. Ewers, D.S. Ellsworth, K.V.R. Schäfer \& R. Oren. 2000. Influence of soil porosity on water use in Pinus taeda. Oecologia 124: 495-505.

Holdridge, L.R. 1978. Ecología basada en zonas de vida. IICA, San José, Costa Rica.

Huston, M. 1980. Soil nutrients and tree species richness in Costa Rican forests. J. Biogeogr. 7: 147-157.

Janzen, D.H. 1986. Tropical dry forest: the most endangered major tropical ecosystem, p. 130-137. In E.O. Wilson (ed.). Biodiversity. National Academic, Washington DC, EEUU.

Janzen, D.H. 2000. Costa Rica's Área de Conservación Guanacaste: a long march to survival through nondamaging biodevelopment. Biodiversity 1: 7-20.

Jaramillo, V.J. \& R.L. Sanford. 1995. Nutrient cycling in tropical deciduous forests, p. 346-361. In S.H Bullock, H.A. Mooney \& E. Medina (eds.). Seasonally dry tropical forests. Cambridge University, Cambridge, Reino Unido.

Jordan, C.F. 1985. Nutrient cycling in tropical forest ecosystems. Wiley, Londres, Reino Unido. 
Kramer, E.A. 1997. Measuring landscape changes in remnant tropical dry forest, p. 386-399. In W.F. Lawrence \& R.G. Bierregaard (eds.). Tropical forest remnants: ecology, management, and conservation of fragmented communities. Chicago University, Chicago, EEUU.

Kussmaul, S. 2000. Estratigrafía de las rocas ígneas, p. 63-86. In P. Denyer \& S. Kussmaul (eds.). Geología de Costa Rica. Tecnológica, Cartago, Costa Rica.

Lathwell, D.J. \& T.L. Grove. 1986. Soil-plant relationships in the tropics. Ann. Rev. Ecol. Syst. 17: 1-16.

Maas, J.M., P. Balvanera, A. Castillo, G.C. Daily, H.A. Mooney, P. Ehrlich, M. Quesada, A. Miranda, V.J. Jaramillo, F. García-Oliva, A. Martínez-Yrizar, H. Cotler, J. López-Blanco, A. Pérez-Jiménez, A. Búrquez, C. Tinoco, G. Ceballos, L. Barraza, R. Ayala \& J.

Mata. 1982. Variaciones pedogenéticas en tres secuencias topográficas del Pacífico seco de Costa Rica. Tesis de Maestría, CATIE, Turrialba, Costa Rica.

Medina, E. 1995. Diversity of life forms of higher plants in neotropical dry forest, p. 221-242. In S.H Bullock, H.A. Mooney \& E. Medina (eds.). Seasonally dry tropical forests. Cambridge University, Cambridge, Reino Unido.

Mesquita, R.C.G., K. Ickes, G. Ganade \& G.B. Williamson. 2001. Alternative successional pathways in the Amazon Basin. J. Ecol. 89: 528-537.

Mulkey, S.S, R.L. Chazdon \& A.P. Smith. 1996. Tropical forest plant ecophysiology. Chapman \& Hall, Nueva York, EEUU.

Murphy, P.G. \& A.E. Lugo. 1986. Ecology of tropical dry forest. Ann. Rev. Ecol. Syst. 17: 67-88.
Nelson, D.W. \& L.E. Sommers. 1982. Total carbon, organic carbon, and organic matter, p. 539-579. In A.L. Page, R.H. Miller \& D.R. Keeney (eds.). Method of soil analysis -part 2- chemical and microbiological properties. ASA, Madison, Wisconsin, EEUU.

Raich, J.W. 1980. Fine roots re-grow rapidly after forest felling. Biotropica 12: 231-232.

Ruiz, J., M.C. Fandiño \& R.L. Chazdon. 2005. Vegetation structure, composition, and species richness across a 56 year chronosequence of dry tropical forest on Providencia Island, Colombia. Biotropica 37: 520-530.

Russell, A.E., J.W. Raich, O.J. Valverde-Barrantes \& R.F. Fisher. 2007. Tree species effects on soil properties in experimental plantations in tropical moist forest. Soil Sci. Soc. Am. J. 71: 1389-1397.

Sanford, R.L. \& E. Cuevas. 1996. Root growth and rhizosphere interactions in tropical forests, p. 268-300. In S.S Mulkey, R.L. Chazdon \& A.P. Smith (eds.). Tropical forest plant ecophysiology. Chapman \& Hall, Nueva York, EEUU.

Soil Survey Staff, 2007. Keys to Soil Taxonomy. USDANRCS, EEUU.

StatSoft Inc. 2000. Statistica 5.5 for Windows [Computer program manual]. StatSoft, Tulsa, Oklahoma, EEUU.

Winters, A.F. 1997. Soils on the Pleistocene ignimbrite Bagaces formation, Guanacaste, Costa Rica: a report on the results of two theses. Wageningen Agricultural University, Wageningen, Holanda.

Zar, J.H. 1999. Biostatistical analysis. Prentice-Hall, Nueva Jersey, EEUU.

Zinke, P.J. 1962. The pattern of influence of individual forest trees on soil properties. Ecology 43: 130-133. 